\title{
Pitfalls in the care of the very sick psychotic patient
}

In the state psychiatric sector consultants are often faced with management of the very sick psychotic patient. There are guidelines to managing treatment resistance in schizophrenia, which may follow the following path.'

If a treatment effect $130-40 \%$ improvement of positive symptoms on standardised rating scales after a month or two of treatment) is not observed, most clinicians switch to a second-generation antipsychotic drug. When switching from either conventional antipsychotics to a second-generation antipsychotic or from one second-generation antipsychotic to another, clinicians will frequently cross-titrate. In this situation one may become trapped in cross-titration (if the patient improves in the middle of cross-titration, the clinician may decide to continue both drugs rather than complete the switch). This type of polypharmacy is not justified. Current treatment guidelines recommend that only after several failures with sequential monotherapies, including consideration of clozapine and convential antipsychotics, should long-term polypharmacy with two antipsychotics be given.

Audits of antipsychotic use in clinical practice suggest that up to $25 \%$ of outpatients and up to $50 \%$ of inpatients take two antipsychotics for long-term maintenance treatment. This is one of the most practised and least investigated phenomena in clinical psychopharmacology.

In the study by Koen et $a l^{2}$ on amisulpiride as adjunct to clozapine in treatment-resistant schizophrenia and schizoaffective disorder, it is suggested that to improve efficacy, $D_{2}$ blockade is increased by adding amisulpiride to treatment with clozapine. It is suggested that addition of amisulpiride to a stable treatment regimen with clozapine may offer additional benefits in terms of negative and depressive symptoms. It is to be hoped that studies like this will lead to double-blind placebo-controlled comparison studies that will guide us scientifically in the management of the difficult psychotic patient. We must remember that current treatment guidelines suggest that maintenance of patients on two antipsychotics, or even very high doses of secondgeneration antipsychotics, should be done with caution, only when all else fails, and only when clearly demonstrated to be beneficial.
The side-effect profiles of conventional and second-generation antipsychotics differ dramatically. Terms such as metabolic syndrome and type 2 diabetes have become commonplace in psychiatric journals of the past 5 - 10 years, whereas modern journals and textbooks do not discuss the side-effects of the conventional antipsychotics, such as anticholinergic side-effects, in much detail. It is assumed that all psychiatrists will use second-generation antipsychotics. This is not the case, especially in the state sector where the difficult-to-treat psychotic patient usually lands up.

As the science of medicine progresses, unfamiliar terminology and conditions are making their appearance in many fields of medicine. A chronic psychotic patient with an acute distended abdomen was recently referred from the hospital where I work to the Department of Surgery after the registrar had diagnosed possible faecal impaction. A diagnosis of abdominal compartment syndrome (ACS) was made at autopsy. This made me rush to a surgery journal.

Classic features of ACS include tachycardia, low blood pressure, respiratory distress, oliguria, abdominal distension, an elevated diaphragm on the chest radiograph, and haemodynamic collapse. ${ }^{3}$ In our patient's case these symptoms were caused by extreme dilatation of the impacted colon, leading to intra-abdominal hypertension and hence ACS. This patient had been treated with combination pharmacotherapy with definite anticholinergic properties, which had caused constipation and later an impacted colon.

In a 1978 textbook ${ }^{4}$ f found the following description of the autonomic effects of psychotropic drugs and how they can cause constipation, a common side-effect of the drugs we use in everyday practice that may have deadly consequences: 'Both anticholinergic activity and the increased availability of norepinephrine at peripheral receptor sites may cause decreased gastrointestinal motility and constipation. Reduction in intestinal motility and secretions may lead to faecal impaction. Constipation is common in psychiatric patients even without drug treatment, but it is frequently reported in association with medications having potent anticholinergic activity (e.g. tricyclic antidepressants, aliphatic and piperidine phenothiazines). Constipation appears to be less frequent 
with the buterophenones, piperazine phenothiazines and MAO inhibitors, and least frequent with the thioxantherines and benzodiazepines.

'Again clinical experience illustrates the complexity of assessing the role of psychoactive agents in producing constipation. The symptom of constipation may improve in depressed patients treated with antidepressants and recently observed has been improvement in constipation in patients treated with lorazapam for anxiety associated with gastrointestinal complaints.

'Constipation may progress to adynamic ileus particularly in elderly patients, in those treated with a combination of drugs with anticholinergic properties and in patients with hypothyroidism. Chronic drug-induced constipation may lead to dilation of the intestine.'

The article by Seller et al. ${ }^{5}$ made me realise that fatal intestinal obstruction in psychiatric patients may be much more common than we think. I wholeheartedly agree with their conclusions that all mental health care providers should have an increased awareness of the risk and possible sequelae of constipation in institutionalised patients, and that there needs to be a low threshold for investigating and treating clozapine-induced constipation.

\section{J L Roos}

\section{Head: Department of Psychiatry}

University of Pretoria

1. Stahl SM. Essential Psychopharmatology Neuroscientific Basis and Practical Applications. 2nd ed. Cambridge: Cambridge University Press, 2002.

2. Koen L, Niehaus DJ, Schutte A, Muller J, Emsley R, Oosthuizen P. Amisulpiride as adjustment to clozapine in treatment-resistant schizophrenia and schizoaffective disorder: An open-label pilot study. South African Journal of Psychiatry 2006; 12: 17-20 (this issue).

3. Schein M, Wirtmann DH, Aprahamian C, Condon RE. The abdominal compartment syndrome. J Am Coll Surg 1995; 180: 745-753.

4. Lipton MA, DiMascio A, Killam KF, eds. Psychopharmacology. A Generation of Progress. New York: Raven Press, 1978.

5. Seller C, Koen L, Niehaus DJH. Clozapine-induced intestinal obstruction - a critical examination of four cases. South African Journal of Psychiatry 2006; 12: $21-24$ (this issue). 